\title{
Fuzzy differential equation with nonlocal conditions and fuzzy semigroup
}

\section{Said Melliani", El Hassan Eljaoui and Lalla Saadia Chadli}

\section{"Correspondence:}

said.melliani@gmail.com

Laboratoire de Mathématiques

Appliquées \& Calcul Scientifique,

Sultan Moulay Slimane University,

BP 523, Beni Mellal, 23000, Morocco

\begin{abstract}
In this work, we use the fuzzy strongly continuous semigroup theory to prove the existence, uniqueness, and some properties of solutions of fuzzy differential equations with nonlocal conditions.
\end{abstract}

Keywords: fuzzy semigroup; generator of a fuzzy semigroup; fuzzy neutral differential equation; nonlocal conditions; mild solutions

\section{Introduction}

Ezzinbi and $\mathrm{Fu}[1]$ studied the neutral differential equation with nonlocal conditions

$$
\begin{aligned}
& \frac{d}{d t}\left[x(t)-F\left(t, x\left(h_{1}(t)\right)\right)\right]=A\left[x(t)-F\left(t, x\left(h_{1}(t)\right)\right)\right]+G\left(t, x\left(h_{2}(t)\right)\right), \\
& x(0)+g(x)=x_{0},
\end{aligned}
$$

where $F, G: I(\subset \mathbb{R}) \times X \rightarrow X$ (Banach space), $h_{1}, h_{2}: I \rightarrow I$, and $A$ is the infinitesimal generator of a strongly continuous semigroup.

Park et al. [2] studied the following fuzzy differential equation:

$$
x^{\prime}(t)=f(t, x(t)), \quad x(0)-g\left(t_{1}, t_{2}, \ldots, t_{p}, x\right)=x_{0},
$$

on $I=[0, a]$, where $f: I \times E^{n} \rightarrow E^{n}$ and $g: I^{p} \times E^{n} \rightarrow E^{n}$ are fuzzy level-wise continuous functions.

Jeong [3] studied the same problem, provided that $f: I \times L_{2} \rightarrow L_{2}$ and $g: I^{p} \times L_{2} \rightarrow L_{2}$ are fuzzy mean-square continuous functions. Balachandran and Chandrasekaran [4] proved the existence and uniqueness of the solutions of a fuzzy delay differential equation with nonlocal conditions. Balasubramaniam and Muralisankar [5] studied the neutral problem

$$
\frac{d}{d t}\left[x(t)-f\left(t, x_{t}\right)\right]=A x(t)+g\left(t, x_{t}\right), \quad x(t)=\psi(t),
$$

on $J=[0, T]$, where $f, g: J \times E^{n} \rightarrow E^{n}$ are fuzzy level-wise continuous functions, and $A$ is a fuzzy coefficient.

(c) 2016 Melliani et al. This article is distributed under the terms of the Creative Commons Attribution 4.0 International License (http://creativecommons.org/licenses/by/4.0/), which permits unrestricted use, distribution, and reproduction in any medium, provided you give appropriate credit to the original author(s) and the source, provide a link to the Creative Commons license, and indicate if changes were made. 
In this paper, we prove the existence and uniqueness of mild solutions for the following fuzzy differential equations with nonlocal conditions:

$$
\left\{\begin{array}{l}
\frac{d x(t)}{d t}=A x(t)+f(t, x(h(t))), \\
x(0)=x_{0}+(-1) g(x),
\end{array}\right.
$$

provided that $x_{0} \in E^{n}, f:[0, a] \times E^{n} \rightarrow E^{n}$ is continuous and satisfies Lipschitz condition, $h:[0, a] \rightarrow[0, a]$ is continuous, and $A$ is the generator of a strongly continuous fuzzy semigroup.

The remainder of this work is organized as follows. Section 2 deals with some preliminaries about fuzzy numbers and fuzzy semigroups. In Section 3, we give sufficient conditions for the existence and uniqueness of a mild solution of the fuzzy differential equation with nonlocal condition (1). In Section 4, we study the continuous dependence between mild solutions and initial data. The last section is devoted to a study of a particular case.

\section{Preliminaries}

\subsection{Fuzzy sets and numbers}

Let $\mathcal{P}_{K}\left(\mathbb{R}^{n}\right)$ denote the family of all nonempty compact convex subsets of $\mathbb{R}^{n}$ and define the addition and scalar multiplication in $\mathcal{P}_{K}\left(\mathbb{R}^{n}\right)$ as usual. Let $A$ and $B$ be two nonempty bounded subsets of $\mathbb{R}^{n}$. The distance between $A$ and $B$ is defined by the Hausdorff metric

$$
d(A, B)=\max \left\{\sup _{a \in A} \inf _{b \in B}\|a-b\|, \sup _{b \in B} \inf _{a \in A}\|a-b\|\right\},
$$

where $\|\cdot\|$ denotes the usual Euclidean norm in $\mathbb{R}^{n}$. Then it is clear that $\left(\mathcal{P}_{K}\left(\mathbb{R}^{n}\right), d\right)$ becomes a complete and separable metric space (see [6]). Denote

$$
E^{n}=\left\{u: \mathbb{R}^{n} \rightarrow[0,1] \mid u \text { satisfies (i)-(iv) below }\right\},
$$

where

(i) $u$ is normal, that is, there exists $x_{0} \in \mathbb{R}^{n}$ such that $u\left(x_{0}\right)=1$;

(ii) $u$ is fuzzy convex;

(iii) $u$ is upper semicontinuous;

(iv) $[u]^{0}=\operatorname{cl}\left\{x \in \mathbb{R}^{n} / u(x)>0\right\}$ is compact.

For $0<\alpha \leq 1$, denote $[u]^{\alpha}=\left\{t \in \mathbb{R}^{n} / u(t) \geq \alpha\right\}$. Then from (i)-(iv) it follows that the $\alpha$-level set $[u]^{\alpha} \in \mathcal{P}_{K}\left(\mathbb{R}^{n}\right)$ for all $0 \leq \alpha \leq 1$.

According to Zadeh's extension principle, we have addition and scalar multiplication in fuzzy number space $E^{n}$ as follows:

$$
[u+v]^{\alpha}=[u]^{\alpha}+[v]^{\alpha}, \quad[k u]^{\alpha}=k[u]^{\alpha},
$$

where $u, v \in E^{n}, k \in \mathbb{R}$, and $0 \leq \alpha \leq 1$.

Define the mapping $D: E^{n} \times E^{n} \rightarrow \mathbb{R}^{+}$as follows:

$$
D(u, v)=\sup _{0 \leq \alpha \leq 1} d\left([u]^{\alpha},[v]^{\alpha}\right),
$$

where $d$ is the Hausdorff metric for nonempty compact sets in $\mathbb{R}^{n}$. Then it is easy to see that $D$ is a metric in $E^{n}$. Using the results in [6], we know that 
(1) $\left(E^{n}, D\right)$ is a complete metric space;

(2) $D(u+w, v+w)=D(u, v)$ for all $u, v, x \in E^{n}$;

(3) $D(k u, k v)=|k| D(u, v)$ for all $u, v \in E^{n}$ and $k \in \mathbb{R}$;

(4) $D(u+v, w+e) \leq D(u, w)+D(v, e)$ for all $u, v, w, e \in E^{n}$.

Also, the following result is known.

\section{Theorem 1 (see [7])}

(i) $u+v=v+u, u+(v+w)=(u+v)+w$;

(ii) if we denote $\tilde{0}=\chi_{\{0\}}$, then $u+\tilde{0}=\tilde{0}+u=u$ for any $u \in E^{n}$;

(iii) with respect to $\tilde{0}$, none of $u \in E^{n} \backslash \mathbb{R}^{n}$ has an opposite member in $E^{n}$;

(iv) for any $a, b \in \mathbb{R}$ with $a, b \geq 0$ or $a, b \leq 0$ and $u \in E^{n}$, we have $(a+b) u=a u+b u$; for general $a, b \in \mathbb{R}$, this property does not hold.

Remark 1 On $E^{n}$, we can define the Hukuhara difference (H-difference) as follows: $u-v$ has sense if there exists $w \in E^{n}$ such that $u=v+w$. Clearly, $u-v$ does not exist for all $u, v \in E^{n}$ (for example, $\tilde{0}-v$ does not exists if $v \neq \tilde{0}$ ).

For short, we can write $u-v$ instead of $u+(-1) v$ for all $u, v \in E^{n}$.

In what follows, we consider $\mathcal{C}_{a}=\mathcal{C}\left([0, a], E^{n}\right)$, the space of all continuous fuzzy functions defined on $[0, a] \subset \mathbb{R}$ into $E^{n}$, where $a>0$. For $u, v \in \mathcal{C}_{a}$, we define the metric

$$
H(u, v)=\sup _{t \in[0, a]} D(u(t), v(t))
$$

Then $\left(\mathcal{C}_{a}, H\right)$ is a complete metric space.

Let $T=[c, d] \subset \mathbb{R}$ be a compact interval. We recall some measurability and integrability properties for the fuzzy set-valued mappings in [8], pp.35, 37.

Definition 1 A mapping $F: T \rightarrow E^{n}$ is strongly measurable if, for all $\alpha \in[0,1]$, the setvalued function $F_{\alpha}: T \rightarrow \mathcal{P}_{K}\left(\mathbb{R}^{n}\right)$ defined by $F_{\alpha}(t)=[F(t)]^{\alpha}$ is Lebesgue measurable when $\mathcal{P}_{K}\left(\mathbb{R}^{n}\right)$ is endowed with the topology generated by the Hausdorff metric $d$.

A mapping $F: T \rightarrow E^{n}$ is called integrably bounded if there exists an integrable function $k: T \rightarrow \mathbb{R}_{+}$such that $D\left(F_{0}(t), \chi_{\{0\}}\right) \leq k(t)$ for all $t \in T$.

Definition 2 Let $F: T \rightarrow E^{n}$. Then the integral of $F$ over $T$, denoted by $\int_{T} F(t) d t$ or $\int_{c}^{d} F(t) d t$, is defined by the equation

$$
\begin{aligned}
{\left[\int_{T} F(t) d t\right]^{\alpha} } & =\int_{T} F_{\alpha}(t) d t \\
& =\left\{\int_{T} f(t) d t / f: T \rightarrow \mathbb{R}^{n} \text { is a measurable selection for } F_{\alpha}\right\}
\end{aligned}
$$

for all $\alpha \in] 0,1]$.

Also, a strongly measurable and integrably bounded mapping $F: T \rightarrow E^{n}$ is said to be integrable over $T$ if $\int_{T} F(t) d t \in E^{n}$.

Proposition 1 ([8]) If $F: T \rightarrow E^{n}$ is strongly measurable and integrably bounded, then $F$ is integrable. 
The following definitions and theorems are given in [9].

Proposition 2 Let $F, G: T \rightarrow E^{n}$ be integrable, and $\lambda \in \mathbb{R}$. Then

(i) $\int_{T}(F(t)+G(t)) d t=\int_{T} F(t) d t+\int_{T} G(t) d t$;

(ii) $\int_{T} \lambda F(t) d t=\lambda \int_{T} F(t) d t$;

(iii) $D(F, G)$ is integrable;

(iv) $D\left(\int_{T} F(t) d t, \int_{T} G(t) d t\right) \leq \int_{T} D(F, G)(t) d t$.

Definition 3 A mapping $F: T \rightarrow E^{n}$ is Hukuhara differentiable at $t_{0} \in T$ if there exists $F^{\prime}(t) \in E^{n}$ such that the limits

$$
\lim _{h \rightarrow 0^{+}} \frac{F(t+h) \ominus F(t)}{h} \text { and } \lim _{h \rightarrow 0^{+}} \frac{F(t) \ominus F(t-h)}{h}
$$

exist and are equal to $F^{\prime}(t)$.

Here the limit is taken in the metric space $\left(E^{n}, D\right)$. At the end points of $T$, we consider only one-sided derivatives.

\subsection{Fuzzy strongly continuous semigroups}

Theorem 2 (Embedding theorem) There exists a real Banach space $X$ such that $E^{n}$ can be embedded as a convex cone $C$ with vertex 0 in $X$. Furthermore, the following conditions hold:

(i) the embedding $j$ is isometric;

(ii) the addition in $X$ induces the addition in $E^{n}$;

(iii) the multiplication by a nonnegative real number in $X$ induces the corresponding operation in $E^{n}$;

(iv) $C-C=\left\{a-b / a, b \in E^{n}\right\}$ is dense in $X$;

(v) $C$ is closed.

Remark 2 As in [10], we can introduce another embedding by the formula $\tilde{j}: E^{n} \rightarrow X$ with $\tilde{j}(u)=j((-1) u), u \in E^{n}$. It has the following properties:

(i) $\|\tilde{j}(u)-\tilde{j}(v)\|=\|j((-1) u)-j((-1) v)\|=D((-1) u,(-1) v)=D(u, v)$;

(ii) $\tilde{j}\left(E^{n}\right)=j\left(E^{n}\right)=C$ since $(-1) E^{n}=E^{n}$;

(iii) for $t, s \geq 0$ and $u, v \in E^{n}$, we have

$$
\begin{aligned}
\tilde{j}(t u+s v) & =j((-1)(t u+s v))=j[t(-1) u+s(-1) v] \\
& =t j((-1) u)+s j((-1) v)=t \tilde{j}(u)+s \tilde{j}(v) .
\end{aligned}
$$

Definition 4 By a fuzzy (one-parameter strongly continuous nonlinear) semigroup on $E^{n}$ we mean a family $\{T(t), t \geq 0\}$ of operators from $E^{n}$ into itself satisfying the following conditions:

(i) $T(0)=I$, the identity mapping on $E^{n}$;

(ii) $T(t+s)=T(t) T(s)$ for all $t, s \geq 0$;

(iii) the function $g:\left[0, \infty\left[\rightarrow E^{n}\right.\right.$ defined by $g(t)=T(t)(x)$ is continuous at $t=0$ for all $x \in E^{n}$, that is,

$$
\lim _{t \rightarrow 0^{+}} T(t)(x)=x
$$


(iv) there exist two constants $M>0$ and $\omega$ such that

$$
D(T(t) x, T(t) y) \leq M e^{\omega t} D(x, y) \quad \text { for } t \geq 0, x, y \in E^{n} .
$$

$\{T(t), t \geq 0\}$ is also called a fuzzy $\mathcal{C}^{0}$-semigroup.

In particular, if $M=1$ and $\omega=0$, we say that $\{T(t), t \geq 0\}$ is a contraction fuzzy semigroup.

Remark 3 Condition (iii) implies that the function $g(t)=T(t)(x)$ is continuous on $[0, \infty[$ for all $x \in E^{n}$.

\section{Remark 4}

- Taking $t=0$ in (iv), we can easily see that $M \geq 1$.

- The quantity $\omega_{0}=\inf \{\omega \in \mathbb{R} \cup\{-\infty\} / \omega$ satisfied (iv) $\}$ is called the type of the fuzzy semigroup.

In the sequel we can choose $\omega>0$.

Definition 5 Let $\{T(t), t \geq 0\}$ be a fuzzy $\mathcal{C}^{0}$-semigroup on $E^{n}$, and $x \in E^{n}$. If for $h>0$ sufficiently small, the Hukuhara difference $T(h) x \ominus x$ exists, then we define

$$
A x=\lim _{h \rightarrow 0^{+}} \frac{T(h) x \ominus x}{h}
$$

whenever this limit exists in the metric space $\left(E^{n}, D\right)$. Then the operator $A: x \mapsto A x$ defined on

$$
D(A)=\left\{x \in E^{n} / \lim _{h \rightarrow 0^{+}} \frac{T(h) x \ominus x}{h} \text { exists }\right\} \subset E^{n}
$$

is called the infinitesimal generator of the fuzzy semigroup $\{T(t), t \geq 0\}$.

Remark 5 The infinitesimal generator $A$ of a fuzzy semigroup $\{T(t), t \geq 0\}$ is unique.

Lemma 1 Let $A$ be the generator of a fuzzy semigroup $\{T(t), t \geq 0\}$ on $E^{n}$. Then for all $x \in E^{n}$ such that $T(t) x \in D(A)$ for all $t \geq 0$, the mapping $t \rightarrow g(t)=T(t) x$ is differentiable, and

$$
g^{\prime}(t)=A T(t) x, \quad \text { that is, } \quad \frac{d}{d t}(T(t) x)=A T(t) x, \quad \forall t \geq 0 .
$$

Remark 6 In the linear case, we have

$$
\frac{d}{d t}(T(t) x)=A T(t) x=T(t) A x, \quad \forall t \geq 0
$$

but in the general (fuzzy) case, $A T(t) \neq T(t) A$. 


\section{Fuzzy differential equation with nonlocal condition}

We consider the fuzzy neutral differential equation with nonlocal conditions

$$
\left\{\begin{array}{l}
\frac{d x(t)}{d t}=A x(t)+f(t, x(h(t))), \\
x(0)=x_{0}+(-1) g(x),
\end{array}\right.
$$

where $A$ generates a strongly continuous fuzzy semigroup $\{T(t), t \geq 0\}$ on $E^{n}, x_{0} \in E^{n}$, $f:[0, a] \times E^{n} \rightarrow E^{n}$, and $h:[0, a] \rightarrow[0, a]$ is a function satisfying some conditions to be described later.

We denote $C_{a}=\mathcal{C}\left([0, a], E^{n}\right)$ and assume that:

$\left(\mathrm{H}_{0}\right) A$ is the infinitesimal generator of a strongly continuous fuzzy semigroup $\{T(t), t \geq 0\}$ on $E^{n}$ such that $D(A)=E^{n}$;

$\left(\mathrm{H}_{1}\right) f:[0, a] \times E^{n} \rightarrow E^{n}$ is continuous and Lipschitzian with respect to the second argument, that is, there exists a constant $L>0$ such that

$$
D(f(t, x), f(t, y)) \leq L D(x, y) \quad \text { for all } t \in[0, a], x, y \in E^{n}
$$

$\left(\mathrm{H}_{2}\right) g: C_{a}=\mathcal{C}\left([0, a], E^{n}\right) \rightarrow E^{n}$ is Lipschitzian, that is, there exists a constant $l>0$ such that

$$
H(g(u), g(v)) \leq l H(u, v), \quad u, v \in C_{a} ;
$$

$\left(\mathrm{H}_{3}\right) h:[0, a] \rightarrow[0, a]$ is continuous;

$\left(\mathrm{H}_{4}\right)$ There exists $M \geq 1$ such that

$$
D(T(t) x, T(t) y) \leq M D(x, y) \quad \text { for } t \geq 0, x, y \in E^{n}
$$

Definition 6 We say that $x$ is a mild solution of Eq. (1) if

(i) $x \in \mathcal{C}\left([0, a], E^{n}\right), x(t) \in D(A)$ for all $t \in[0, a]$; and

(ii) $x(t)=T(t)\left[x_{0}+(-1) g(x)\right]+\int_{0}^{t} T(t-s) f(s, x(h(s))) d s$ for all $t \in[0, a]$.

Theorem 3 Suppose that assumptions $\left(\mathrm{H}_{0}\right)-\left(\mathrm{H}_{4}\right)$ hold. Then for any $x_{0} \in E^{n}$, Eq. (1) has a unique mild solution, provided that

$$
L_{0}=M(l+a L)<1 .
$$

Proof We define the mapping $P: C_{a} \rightarrow C_{a}$ by

$$
P x(t)=T(t)\left[x_{0}+(-1) g(x)\right]+\int_{0}^{t} T(t-s) f(s, x(h(s))) d s
$$

for all $x \in C_{a}$ and $t \in[0, a]$.

Step 1. For $x \in C_{a}, t \in[0, a[$, and $\xi>0$ sufficiently small,

$$
\begin{aligned}
& D(P x(t+\xi), P x(t)) \\
& \quad=D\left(T(t) T(\xi)\left[x_{0}+(-1) g(x)\right]+\int_{0}^{t+\xi} T(t+\xi-s) f(s, x(h(s))) d s,\right.
\end{aligned}
$$




$$
\begin{aligned}
& \left.T(t)\left[x_{0}+(-1) g(x)\right]+\int_{0}^{t} T(t-s) f(s, x(h(s))) d s\right) \\
\leq & D\left(T(t) T(\xi)\left[x_{0}+(-1) g(x)\right], T(t)\left[x_{0}+(-1) g(x)\right]\right) \\
& +D\left(\int_{0}^{\xi} T(t+\xi-s) f(s, x(h(s))) d s, \hat{0}\right) \\
& +D\left(\int_{\xi}^{t+\xi} T(t+\xi-s) f(s, x(h(s))) d s, \int_{0}^{t} T(t-s) f(s, x(h(s))) d s\right) .
\end{aligned}
$$

By changing the variable we have

$$
\int_{\xi}^{t+\xi} T(t+\xi-s) f(s, x(h(s))) d s=\int_{0}^{t} T(t-s) f(s+\xi, x(h(s+\xi))) d s .
$$

Therefore,

$$
\begin{aligned}
D(P x( & (t \xi), P x(t)) \\
\leq & M D\left(T(\xi)\left[x_{0}+(-1) g(x)\right],\left[x_{0}+(-1) g(x)\right]\right) \\
\quad & D\left(\int_{0}^{\xi} T(t+\xi-s) f(s, x(h(s))) d s, \hat{0}\right) \\
\quad & +D\left(\int_{0}^{t} T(t-s) f(s+\xi, x(h(s+\xi))) d s, \int_{0}^{t} T(t-s) f(s, x(h(s))) d s\right) \\
\leq & M D\left(T(\xi)\left[x_{0}+(-1) g(x)\right],\left[x_{0}+(-1) g(x)\right]\right) \\
& +\int_{0}^{\xi} D(T(t+\xi-s) f(s, x(h(s))), \hat{0}) d s \\
& +\int_{0}^{t} D(T(t-s) f(s+\xi, x(h(s+\xi))), T(t-s) f(s, x(h(s)))) d s \\
\leq & M D\left(T(\xi)\left[x_{0}+(-1) g(x)\right],\left[x_{0}+(-1) g(x)\right]\right) \\
& +\int_{0}^{\xi} D(T(t+\xi-s) f(s, x(h(s))), \hat{0}) d s \\
& +M \int_{0}^{t} D(f(s+\xi, x(h(s+\xi))), f(s, x(h(s)))) d s .
\end{aligned}
$$

Using assumptions $\left(\mathrm{H}_{0}\right)-\left(\mathrm{H}_{4}\right)$, we can easily show that

$$
D\left(T(\xi)\left[x_{0}+(-1) g(x)\right],\left[x_{0}+(-1) g(x)\right]\right) \rightarrow 0 \quad \text { as } \xi \rightarrow 0^{+}
$$

and

$$
\int_{0}^{\xi} D(T(t+\xi-s) f(s, x(h(s))), \hat{0}) d s \rightarrow 0 \quad \text { as } \xi \rightarrow 0^{+} .
$$

By the dominated convergence theorem we have

$$
\int_{0}^{t} D(f(s+\xi, x(h(s+\xi))), f(s, x(h(s)))) d s \rightarrow 0 \quad \text { as } \xi \rightarrow 0^{+} .
$$

Then, $D(P x(t+\xi), P x(t)) \rightarrow 0$ as $\xi \rightarrow 0^{+}$. 
Let us prove that $D(P x(t-\xi), P x(t)) \rightarrow 0$ as $\xi \rightarrow 0^{+}$. For $\left.\left.x \in C_{a}, t \in\right] 0, a\right]$, and $\xi>0$ sufficiently small,

$$
\begin{aligned}
D(P x(t-\xi), P x(t)) \\
=D\left(T(t-\xi)\left[x_{0}+(-1) g(x)\right]+\int_{0}^{t-\xi} T(t-\xi-s) f(s, x(h(s))) d s,\right. \\
\left.\quad T(t-\xi) T(\xi)\left[x_{0}+(-1) g(x)\right]+\int_{0}^{t} T(t-\xi) T(\xi) f(s, x(h(s))) d s\right) \\
\leq D\left(T(t-\xi)\left[x_{0}+(-1) g(x)\right], T(t-\xi) T(\xi)\left[x_{0}+(-1) g(x)\right]\right) \\
\quad+D\left(\int_{0}^{t-\xi} T(t+\xi-s) f(s, x(h(s))) d s, \int_{0}^{t-\xi} T(t-\xi-s) T(\xi) f(s, x(h(s))) d s\right) \\
\quad+D\left(\hat{0}, \int_{t-\xi}^{t} T(t-s) f(s, x(h(s))) d s\right) .
\end{aligned}
$$

Hence,

$$
\begin{aligned}
& D(P x(t-\xi), P x(t)) \\
& \leq M D\left(x_{0}+(-1) g(x), T(\xi)\left[x_{0}+(-1) g(x)\right]\right) \\
& \quad+\int_{t-\xi}^{t} D(\hat{0}, T(t-s) f(s, x(h(s)))) d s \\
& \quad+\int_{0}^{t-\xi} D(T(t+\xi-s) f(s, x(h(s))), T(t-\xi-s) T(\xi) f(s, x(h(s)))) d s \\
& \leq M D\left(T(\xi)\left[x_{0}-g(x)\right], x_{0}-g(x)\right)+\int_{t-\xi}^{t} D(\hat{0}, T(t-s) f(s, x(h(s)))) d s \\
& \quad+M \int_{0}^{t} D(f(s, x(h(s))), T(\xi) f(s, x(h(s)))) d s .
\end{aligned}
$$

Using assumptions $\left(\mathrm{H}_{0}\right)-\left(\mathrm{H}_{4}\right)$, we can easily show that

$$
D\left(T(\xi)\left[x_{0}+(-1) g(x)\right], x_{0}+(-1) g(x)\right) \rightarrow 0 \quad \text { as } \xi \rightarrow 0^{+}
$$

and

$$
\int_{t-\xi}^{t} D(\hat{0}, T(t-s) f(s, x(h(s)))) d s \rightarrow 0 \quad \text { as } \xi \rightarrow 0^{+} .
$$

By the dominated convergence theorem we have

$$
\int_{0}^{t} D(f(s, x(h(s))), T(\xi) f(s, x(h(s)))) d s \rightarrow 0 \quad \text { as } \xi \rightarrow 0^{+} .
$$

Then, $D(P x(t-\xi), P x(t)) \rightarrow 0$ as $\xi \rightarrow 0^{+}$. Consequently, $P x$ is continuous at each $t \in[0, a]$. Hence, $P x \in C_{a}$, that is, $P$ maps $C_{a}$ into itself.

Step 2. Now we will show that $P$ is a strict contraction on $C_{a}$. 
Letting $x, y \in C_{a}$ and $t \in[0, a]$, we have

$$
\begin{aligned}
& D(P x(t), P y(t)) \\
&= D\left(T(t)\left[x_{0}+(-1) g(x)\right]+\int_{0}^{t} T(t-s) f(s, x(h(s))) d s\right. \\
&\left.T(t)\left[x_{0}+(-1) g(y)\right]+\int_{0}^{t} T(t-s) f(s, y(h(s))) d s\right) \\
& \leq D\left(T(t)\left[x_{0}+(-1) g(x)\right], T(t)\left[x_{0}+(-1) g(y)\right]\right) \\
&+\int_{0}^{t} D(T(t-s) f(s, x(h(s))), T(t-s) f(s, y(h(s)))) d s \\
& \leq M D\left(x_{0}+(-1) g(x), x_{0}+(-1) g(y)\right)+M \int_{0}^{t} D(f(s, x(h(s))), f(s, y(h(s)))) d s \\
& \leq M D(g(x), g(y))+M L \int_{0}^{t} D(x(h(s)),(h(s))) d s \\
& \leq M l H(x, y)+a M L H(x, y) \\
& \leq M(l+a L) H(x, y)=L_{0} H(x, y) .
\end{aligned}
$$

Hence,

$$
H(P x, P y)=\sup _{0 \leq t \leq a} D(P x(t), P y(t)) \leq L_{0} H(x, y) .
$$

Since $L_{0}<1, P$ is a contraction, and there exists a unique $x \in C_{a}$ such that $P x=x$.

Hence, $x$ is the unique mild solution of Eq. (1).

\section{Continuous dependence on initial data}

Theorem 4 Suppose that assumptions $\left(\mathrm{H}_{0}\right)-\left(\mathrm{H}_{4}\right)$ and the condition $L_{0}=M(l+a L)<1$ hold. Let $x=x\left(t, x_{0}\right)$ and $y=y\left(t, y_{0}\right)$ be mild solutions of Eq. (1) corresponding to $x_{0}$ and $y_{0}$, respectively. Then

$$
H\left(x\left(t, x_{0}\right), y\left(t, y_{0}\right)\right) \leq \frac{M}{1-L_{0}} D\left(x_{0}, y_{0}\right) .
$$

Proof For all $t \in[0, a]$, we have

$$
\begin{aligned}
& D\left(x\left(t, x_{0}\right), y\left(t, y_{0}\right)\right) \\
&=D\left(T(t)\left[x_{0}+(-1) g(x)\right]+\int_{0}^{t} T(t-s) f(s, x(h(s))) d s,\right. \\
&\left.\quad T(t)\left[y_{0}+(-1) g(y)\right]+\int_{0}^{t} T(t-s) f(s, y(h(s))) d s\right) \\
& \leq D\left(T(t)\left[x_{0}+(-1) g(x)\right], T(t)\left[y_{0}+(-1) g(y)\right]\right) \\
& \quad+\int_{0}^{t} D(T(t-s) f(s, x(h(s))), T(t-s) f(s, y(h(s)))) d s \\
& \leq M D\left(x_{0}+(-1) g(x), y_{0}+(-1) g(y)\right)+M \int_{0}^{t} D(f(s, x(h(s))), f(s, y(h(s)))) d s
\end{aligned}
$$




$$
\begin{aligned}
& \leq M D\left(x_{0}, y_{0}\right)+M D(g(x), g(y))+M L \int_{0}^{t} D(x(h(s)), y(h(s))) d s \\
& \leq M D\left(x_{0}, y_{0}\right)+M l H(x, y)+a M L H(x, y) \\
& \leq M D\left(x_{0}, y_{0}\right)+L_{0} H(x, y) .
\end{aligned}
$$

Hence,

$$
H(x, y)=H\left(x\left(t, x_{0}\right), y\left(t, y_{0}\right)\right) \leq M D\left(x_{0}, y_{0}\right)+L_{0} H(x, y) .
$$

Since $L_{0}<1$, we deduce that

$$
H\left(x\left(t, x_{0}\right), y\left(t, y_{0}\right)\right) \leq \frac{M}{1-L_{0}} D\left(x_{0}, y_{0}\right) .
$$

\section{A particular case}

Now we study the following special equation:

$$
\left\{\begin{array}{l}
\frac{d x(t)}{d t}=A x(t)+f(t, x(t)), \quad t \in[0, a], \\
x(0)=x_{0}+(-1) \sum_{i=1}^{p} g_{i}\left(x\left(t_{i}\right)\right)
\end{array}\right.
$$

where $p \in \mathbb{N}^{*}$ and $0 \leq t_{1}<t_{2}<\cdots<t_{p} \leq a$. We assume that:

$\left(\mathrm{H}_{5}\right)$ there exist constants $M_{0} \geq 1$ and $\omega_{0}>0$ such that

$$
D(T(t) x, T(t) y) \leq M_{0} e^{-\omega_{0} t} D(x, y) \quad \text { for } t \geq 0, x, y \in E^{n},
$$

with

$$
\left(M_{0} L-\omega_{0}\right)<0 \quad \text { and } \quad a M_{0} L<1
$$

$\left(\mathrm{H}_{6}\right) g_{i}: E^{n} \rightarrow E^{n}$ is Lipschitz continuous: there exist constants $k_{i}>0$ such that

$$
D\left(g_{i}(x), g_{i}(y)\right) \leq k_{i} D(x, y), \quad x, y \in E^{n}, i=1,2, \ldots, p .
$$

Theorem 5 Suppose that assumptions $\left(\mathrm{H}_{0}\right),\left(\mathrm{H}_{1}\right),\left(\mathrm{H}_{4}\right),\left(\mathrm{H}_{5}\right)$, and $\left(\mathrm{H}_{6}\right)$ hold. Then for any $x_{0} \in E^{n}$, Eq. (2) has a unique mild solution, provided that

$$
\sum_{i=1}^{p} k_{i} M_{0} \exp \left[\left(M_{0} L-\omega_{0}\right) t_{i}\right]<1 .
$$

Proof Let $v \in E^{n}$. Since $L_{0}<1$, there exists a unique fuzzy continuous function $x(\cdot, v)$ such that

$$
x(t, v)=T(t) v+\int_{0}^{t} T(t-s) f(s, x(s, v)) d s, \quad \forall t \in[0, a] .
$$

Then the mapping $Q: E^{n} \rightarrow E^{n}$ given by the following expression is well defined:

$$
Q v=x(0)-\sum_{i=1}^{p} g_{i}\left(x\left(t_{i}, v\right)\right), \quad v \in E^{n},
$$


where $x(0)=x(0, v)$. For all $u, v \in E^{n}$, we have

$$
\begin{aligned}
D(Q u, Q v) & =D\left(x(0)-\sum_{i=1}^{p} g_{i}\left(x\left(t_{i}, u\right)\right), x(0)-\sum_{i=1}^{p} g_{i}\left(x\left(t_{i}, v\right)\right)\right) \\
& =D\left(\sum_{i=1}^{p} g_{i}\left(x\left(t_{i}, u\right)\right), \sum_{i=1}^{p} g_{i}\left(x\left(t_{i}, v\right)\right)\right) \\
D(Q u, Q v) & \leq \sum_{i=1}^{p} k_{i} D\left(x\left(t_{i}, u\right), x\left(t_{i}, v\right)\right)
\end{aligned}
$$

For every $t \in[0, a]$, we have

$$
\begin{aligned}
& D(x(t, u), x(t, v)) \\
& \quad \leq D(T(t) u, T(t) v)+D\left(\int_{0}^{t} T(t-s) f(s, x(s, u)) d s, \int_{0}^{t} T(t-s) f(s, x(s, v)) d s\right) \\
& \quad \leq M_{0} e^{-\omega_{0} t} D(u, v)+\int_{0}^{t} D(T(t-s) f(s, x(s, u)), T(t-s) f(s, x(s, v))) d s \\
& \quad \leq M_{0} e^{-\omega_{0} t} D(u, v)+M_{0} \int_{0}^{t} e^{-\omega_{0}(t-s)} D(f(s, x(s, u)), f(s, x(s, v))) d s, \\
& D(x(t, u), x(t, v)) \leq M_{0} e^{-\omega_{0} t} D(u, v)+M_{0} L \int_{0}^{t} e^{-\omega_{0}(t-s)} D(x(s, u), x(s, v)) d s .
\end{aligned}
$$

Thus,

$$
e^{\omega_{0} t} D(x(t, u), x(t, v)) \leq M_{0} D(u, v)+M_{0} L \int_{0}^{t} e^{\omega_{0} s} D(x(s, u), x(s, v)) d s .
$$

Using Gronwall's inequality, we deduce that

$$
e^{\omega_{0} t} D(x(t, u), x(t, v)) \leq M_{0} \exp \left(M_{0} L t\right) D(u, v)
$$

It follows that

$$
D(x(t, u), x(t, v)) \leq M_{0} \exp \left(\left(M_{0} L-\omega_{0}\right) t\right) D(u, v) .
$$

So we conclude that

$$
D(Q u, Q v) \leq \sum_{i=1}^{p} k_{i} M_{0} \exp \left[\left(M_{0} L-\omega_{0}\right) t_{i}\right] D(u, v) .
$$

Since $\sum_{i=1}^{p} k_{i} M_{0} \exp \left[\left(M_{0} L-\omega_{0}\right) t_{i}\right]<1, Q$ is a strict contraction on the complete metric space $\left(E^{n}, D\right)$. So, it has a unique fixed point $v \in E^{n}$, that is, $Q v=v$. The corresponding solution $x(\cdot, v)$ is a mild solution of Eq. (2). 
Authors' contributions

All authors contributed equally to the writing of this paper. All authors read and approved the final manuscript.

\section{Acknowledgements}

The authors express their sincere thanks to the anonymous referees for numerous helpful and constructive suggestions which have improved the manuscript.

Received: 27 May 2015 Accepted: 19 January 2016 Published online: 02 February 2016

\section{References}

1. Fu, X, Ezzinbi, K: Existence of solutions for neutral functional differential evolution equations with nonlocal conditions. Nonlinear Anal. 54, 215-227 (2003)

2. Park, JY, Han, HK, Son, KH: Fuzzy differential equation with nonlocal condition. Fuzzy Sets Syst. 115, 365-369 (2000)

3. Jeong, JU: Fuzzy differential equations with nonlocal condition. J. Appl. Math. Comput. 17, 509-517 (2005)

4. Balachandran, $\mathrm{K}$, Chandrasekaran, $\mathrm{K}$ : Existence of solutions of a delay differential equation with nonlocal condition. Indian J. Pure Appl. Math. 27, 443-449 (1996)

5. Balasubramaniam, P, Muralisankar, S: Existence and uniqueness of a fuzzy solution for the nonlinear fuzzy neutral functional differential equation. Comput. Math. Appl. 42, 961-967 (2001)

6. Puri, ML, Ralescu, DA: Fuzzy random variables. J. Math. Anal. Appl. 114, 409-422 (1986)

7. Gal, CG, Gal, SG: Semigroups of operators on spaces of fuzzy-number-valued functions with applications to fuzzy differential equations (2013). arXiv:1306.3928v1

8. Lakshmikantham, V, Gnana Bhaskar, T, Vasundhara Devi, J: Theory of Set Differential Equations in Metric Spaces. Cambridge Scientific Publishers, Cambridge (2006)

9. Kaleva, O: Fuzzy differential equations. Fuzzy Sets Syst. 24, 301-317 (1987)

10. Bede, B, Gal, SG: Generalization of the differentiability of fuzzy-number-valued functions with applications to fuzzy differential equations. Fuzzy Sets Syst. 151, 581-599 (2005)

\section{Submit your manuscript to a SpringerOpen ${ }^{\circ}$ journal and benefit from:}

- Convenient online submission

- Rigorous peer review

- Immediate publication on acceptance

- Open access: articles freely available online

- High visibility within the field

- Retaining the copyright to your article 